\title{
EDITORIAL AND COMMENT \\ How to Deliver Care Management: Guidance from an Analysis of Communication Patterns in Primary Care
}

\author{
Ann S. O'Malley, MD, MPH \\ Mathematica Policy Research, Washington, DC, USA. \\ $\mathrm{J}$ Gen Intern Med 33(5):576-7 \\ DOI: $10.1007 / \mathrm{s} 11606-018-4387-9$ \\ (c) Society of General Internal Medicine 2018
}

$\mathrm{C}$ are management is a patient-centered approach to helping patients and their support systems better manage health conditions. ${ }^{1}$ It includes patient education, goal setting, and self-management support. As part of such efforts, the term "care manager" is often used to refer to the activities of a nurse who works closely with patients and their primary care team to help provide chronic care management and coordination during care transitions.

When implemented in a controlled manner-with rigorous training and adequate resources - care managers' work has the potential to improve chronic condition management and to lower costs. ${ }^{2}$ However, evaluations of care managers in the "real-world" have shown that their effectiveness varies, due in part to wide variation in implementation.

Determining whether care managers improve patient outcomes and are cost-effective first requires a clearer understanding of how to incorporate them into primary care delivery. Key pieces of this puzzle include understanding where to place the care manager (in the practice, in the larger health-care delivery system, or at a health plan); who is best situated to employ and supervise the care manager (health plans, provider organizations, or practice sites); and how to integrate the care manager into the primary care team.

Prior work suggests that embedded care managers are better at improving chronic condition management than care managers who are located outside the practice. ${ }^{3-5}$ A study by Holtrop and colleagues ${ }^{6}$ in this issue of the Journal of General Internal Medicine brings us a step closer to understanding why this might be the case.

Holtrop et al. used social network analysis to gain insight into the communication patterns of primary care teams and care managers across a range of care management structures. Specifically, they sought to answer two questions:

1. Do care managers play a key role in chronic disease management in the practice, as reported by other practice members?

Published online March 12, 2018
2. Does the care manager's level of connectivity in the practice's communication network vary based on the type of care management structure used (that is, whether the care manager is off site, co-located, or embedded in the primary care practice)?

An off-site care manager, according to the study, was one "who did not maintain an office or workspace at the practice and was not an employee of the practice." A co-located care manager was "on site at the practice part time and shared with other practices but was employed by the practice association or practice group." Finally, an embedded care manager was "a full- or part-time employee of the practice [who] maintains a workspace in the practice."

In brief, the answer to both study questions is "yes." Embedded care managers - and co-located care managers, to a lesser extent-were more likely to be at the core of the communication network compared with off-site care managers. They also acted as a "hub for communication" with others in the practice. Moreover, clinicians rated the performance of embedded and co-located care managers more highly than that of off-site care managers.

So what do these findings imply for primary care interventions that include care management?

Primary care interventions, such as medical home models, have a lot of moving parts. Multi-component interventions for chronic condition management generally work better than single-component interventions. ${ }^{4,7}$ But multiple components increase the number of factors that can vary in implementation across sites, including the various ways that care management is structured. This poses a challenge for evaluation. Rigorous experimental and quasi-experimental designs are critical to evaluating multi-component interventions in the real world, but there is also a need for concurrent "micro-studies" of smaller pieces of these interventions. This is particularly important when evidence about how to best implement certain activities in the "real world" is lacking.

Holtrop and colleagues' use of social network analysis applies a microscope to one aspect of such interventions. In doing so, their study brings us closer to understanding how the location and employment models for care managers might be structured to maximize communication between the care manager and the primary care team. It also tells us that clinicians are more likely to rate care managers' performance highly when they are working in the same practice. Thus, clinicians 
and the other members of the practice may be more likely to engage with care managers to enhance patient care.

Besides the questions that Holtrop et al. raise, there are many other aspects of multi-component interventions in primary care delivery that would benefit from close examination. Using care management for chronic conditions in primary care as just one example, what is the ideal training and licensure for care managers, and how does this differ by subpopulations served? In the Holtrop et al. study, the most common type of care managers were nurses with bachelor's degrees, followed by social workers. But, the clinical background and training of a care manager likely varies based on the needs of the patients.

Other micro-studies could address questions such as, what training should primary care clinicians and other members of the primary care team receive to ensure that they engage effectively with care managers? How can health IT tools and electronic health records (EHRs) be modified to maximize communication between members of the primary care team-including care managers, who often use separate care management software that interfaces poorly (if at all) with the practice's EHR?

Many of these questions do not require social network analysis. But alongside other analytic methods, social network analysis is a tool that can inform the design, implementation, and evaluation of interventions, particularly when graphic displays of communication and referral patterns would be useful.

An area ripe for social network analysis in primary carebased care management is how practices are coordinating with community-based social services and behavioral health providers to better meet patients' social, behavioral, and physical health needs. Chronic condition management and premature mortality are as much a function of people's behaviors and social environments as they are of the health-care delivery system. ${ }^{8,9}$ Thus, mapping patients' interactions patterns with behavioral, social support and medical services, as well as mapping how providers from these respective entities share information to care for people, is likely to be beneficial.

Corresponding Author: Ann S. O'Malley, MD, MPH; Mathematica Policy Research, Washington, DC, USA (e-mail: aomalley@mathematicampr.com).

\section{Compliance with Ethical Standards:}

Conflict of Interest: The authors declare that they do not have a conflict of interest.

\section{REFERENCES}

1. Center for Health Care Strategies, Inc. Care management definition and framework. 2007. Available at: http://www.chcs.org/usr_doc/Care_Management_Framework.pdf. Accessed 19 Nov 2013.

2. Hsu J, Price M, Vogeli C, et al. Bending the spending curve by altering care delivery patterns: the role of care management within a pioneer ACO. Health Aff (Millwood). 2017;36(5):876-84.

3. Taliani CA, Bricker PL, Adelman AM, Cronholm PF, Gabbay RA Implementing effective care management in the patient-centered medical home. Am J Manag Care. 2013;19(12):957-64.

4. Coleman K, Mattke S, Perrault PJ, Wagner EH. Untangling practice redesign from disease management: how do we best care for the chronically ill? Ann Rev Public Health. 2009;30:385-408.

5. Peikes D, Chen A, Schore J, Brown R. Effects of care coordination on hospitalization, quality of care, and health care expenditures among Medicare beneficiaries: 15 randomized trials. JAMA. 2009;301(6):603-18.

6. Holtrop JS, Ruland S, Diaz S, Morrato EH, Jones E. Using social network analysis to examine the effect of care management structure on chronic disease management communication within primary care. J Gen Intern Med. https://doi.org/10.1007/s11606-017-4247-z.

7. Bodenheimer T, Wagner EH, Grumbach $\mathbf{K}$. Improving primary care for patients with chronic illness: the chronic care model, part 2. JAMA. 2002;288(15):1909-14.

8. McGinnis JM, Foege WH. Actual causes of death in the United States. JAMA. 1993;270(18):2207-12.

9. Murray CJ, Atkinson C, Bhalla K, et al. The state of U.S. health, 1990 2010: burden of diseases, injuries, and risk factors. JAMA. 2013;310(6):591-608 